\title{
Nutrition Professionals' Knowledge, Consumption and Recommendations of Whole Grains
}

Kristen Hicks-Roof ${ }^{1}$, Tamara Marsh ${ }^{1}$, Krystal Walker ${ }^{1}$, Cristina Reyes Gonzalez ${ }^{1}$, Kelly Toups ${ }^{2}$, Hanadi Hamadi ${ }^{3}$, Jasper Xu ${ }^{3}$

${ }^{1}$ Department of Nutrition and Dietetics, University of North Florida, Jacksonville, FL

2Oldways, Boston MA

${ }^{3}$ Department of Health Administration, University of North Florida, Jacksonville, FL

BACKGRDUND

\section{ARSTRACT}

Research outcome: To determine the relationship between nutrition professionals' knowledge, consumption, and recommendations of whole grains.

Methods: A cross-sectional study was conducted with nutrition professionals $(n=480)$. Participants were recruited via email and social media from October 2020-February 2021. The participants completed a validated 21 -question survey about whole grain knowledge, consumption, and recommendations.

Analysis: The analyses included descriptive statistics and Pearson correlation tests.

Results: Survey respondents included $72.5 \%$ Registered Dietitians Nutritionists (RD/RDN) and 27.5\% RD in training (e.g. dietetic intern, student). Nutrition professionals felt whole grains were important in their own diet $(93 \%)$ and their patient/clients diet $(75 \%)$. A slight majority of nutrition professionals ( $58 \%$ ) strongly or somewhat agreed that "personal grain preference influences the grains that you recommend to patients/clients", despite $67 \%$ agreeing to the statement, "client

income/background influences the types of grains you

recommend to patients/clients." Nutrition professionals correctly classified $14.9 \pm 3.2$ of the 23 food classification questions and $9.6 \pm 1.8$ for the 11 whole grain knowledge statements. Nutrition professionals with direct patient care $(n=373)$ have personally consumed $15.9 \pm 3.74$ different grains and recommended $9 \pm 4.15$ the correlation being $r=0.29$, indicating a significant positive correlation.

Conclusions: Nutrition professionals were able to identify whole grain foods and demonstrated knowledge of whole grain benefits; however, there was a disconnect between knowledge and recommendations. Future research should further investigate nutrition professionals' knowledge, beliefs, and attitudes towards whole grains. To align nutrition professionals' whole grain knowledge and recommendation practices, there is a need for enhanced training and education on whole grain varieties. 2020-2025) 2015) Dietetics) (Edwards 2008; Chase 2003)

\section{METHODS} professionals $(n=480)$. recommendations. whole grains.

\section{RESULTS}

$98 \%$ of Americans do not meet whole grain recommendations (Dietary Guidelines for Americans

- Whole grain consumption has shown a reduced risk of many chronic conditions (e.g. cardiovasulcar disease, type 2 diabetes, and cancer.) (Koo, 2018; Ampatzoglou,

- Registered Dietitian Nutritionists (RD/RDNs) are trained on evidence-based practices (Academy of Nutrition and

- Self-efficacy among dietitians promote whole grains is low

- A cross-sectional study was conducted with nutritional

- Participants were recruited via email and social media platforms from December 2020 to February 2021.

- The participants completed a 21-question validated survey about whole grain knowledge, consumption, and
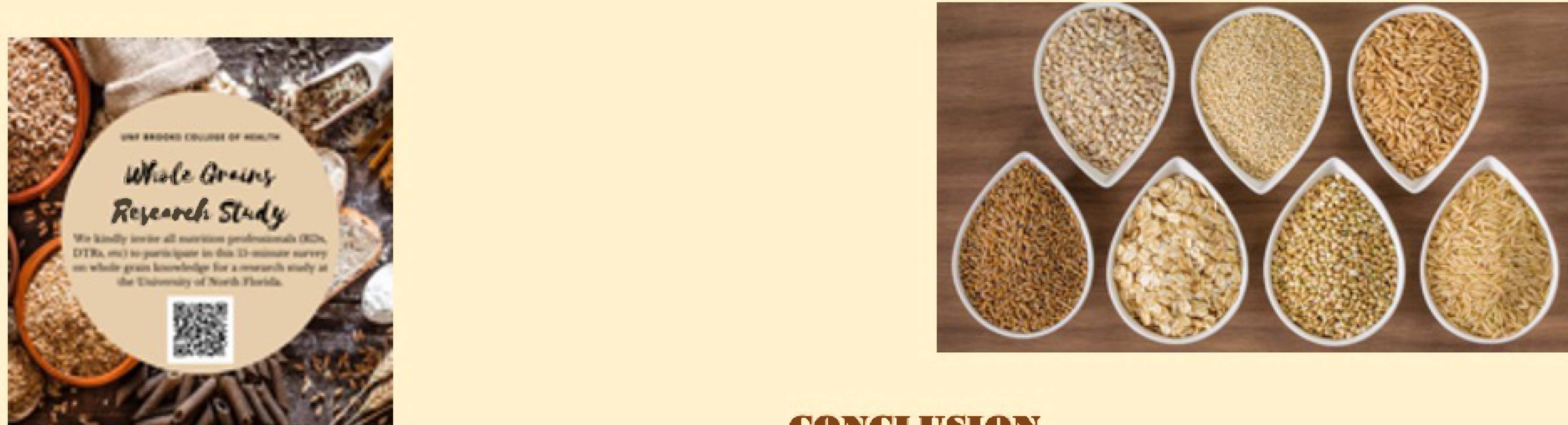

\section{CONCLUSION}

- Nutrition professionals are knowledgeable about whole grains.

- There was a disconnect between nutrition professionals' knowledge and recommendations.

- Future research should further investigate nutrition professionals' knowledge, beliefs, and attitudes towards

There is a need for improved training and education on whole grain varieties to align nutrition professionals whole grain knowledge and recommendation practices 